\title{
Selection of reference genes for normalization of quantitative polymerase chain reaction data in mouse models of heart failure
}

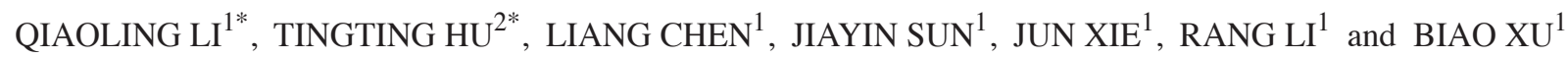 \\ ${ }^{1}$ Department of Cardiology, Nanjing Drum Tower Hospital, The Affiliated Hospital of Nanjing University Medical School, \\ Nanjing, Jiangsu 210008; ${ }^{2}$ Department of Cardiology, Huai'an First People's Hospital, Huai'an, Jiangsu 223300, P.R. China
}

Received November 19, 2013; Accepted July 14, 2014

DOI: $10.3892 / \mathrm{mmr} .2014 .2750$

\begin{abstract}
The accurate measurement of mRNA expression levels is crucially dependent on the use of relevant reference genes for the normalization of data. Currently, heart failure is a serious and widespread disease, and multiple mouse models are utilized for the study of this complex disease. Although mouse models are commonly used to study cardiovascular disease, various studies have not employed the appropriate selection strategies. The present study investigated the expression stability of eight candidate reference genes (GAPDH, ACTB, B2M, CycA, TBP, PBGD, HTRP 1 and 18S) in two mouse models of heart failure, including the transverse aortic arch constriction (TAC) model and the myocardial infarction (MI) model, using GeNorm software. The expression of BNP was normalized using different reference gene strategies, and it was demonstrated that its induction following heart failure was most profound with the optimal reference gene combination. The most stable genes were identified as follows: TBP and CycA in the MI model, and PBGD and GAPDH in the TAC model. The present study provides important information for reference gene selection in mouse models of heart failure, and will aid further investigations of the transcriptome in cardiovascular research.
\end{abstract}

\section{Introduction}

The mouse models of myocardial infarction (MI) and transverse aortic arch constriction (TAC) are widely used in the study of heart failure (HF), for investigating pathophysiological mechanisms and for improving diagnostic and

Correspondence to: Dr Biao Xu, Department of Cardiology, Nanjing Drum Tower Hospital, The Affiliated Hospital of Nanjing University Medical School, 321 Zhong Shan Road, Nanjing 210008, P.R. China

E-mail: xubiao@medmail.com.cn

*Contributed equally

Key words: reference genes, quantitative reverse transcription polymerase chain reaction, heart failure therapeutic approaches (1). Quantitative polymerase chain reaction (qPCR) has become a crucial technique for studying $\mathrm{HF}$, as it possesses the advantages of improved accuracy, sensitivity and specificity, a larger dynamic range and greater speed as compared with other methods. However, no single reference gene (RG) has been identified that is able to maintain stable expression levels under all conditions. It has been previously suggested that the expression levels of previously well characterized RGs, including GAPDH, ACTB and B2M, can vary markedly across cells, tissues and experimental conditions, emphasizing the requirement to adopt alternative RGs and appropriate strategies for their selection (2-4).

Sundaresan et al (5) indicated that the levels of tubulin and actin are variable during heart failure in TAC mice. Previous research has suggested that the most stable RGs vary between the chambers of the heart in the minipig (6). Koyanagi et al (7) demonstrated that CycA expression was the least variable in a hypertension-induced cardiac dysfunction rat model. Another study demonstrated that ACTB was the most stable RG in heart tissue from murine models of cardiometabolic disease (8). This strategy of RG selection has also been applied to the human myocardium (9).

To determine the appropriate RGs under the condition of HF in mice, the current study utilized geNorm software (Biogazelle, Zwijnaarde, Belgium). geNorm is a bioinformatics tool designed to rank candidate RGs using a normalization factor calculated from the expression level of the candidate RGs in an array of given samples (10).

The aim of the present study was to select a set of RGs to be used for normalizing mRNA expression data obtained by qPCR in two HF mouse models. Eight candidate RGs, including GAPDH, ACTB, B2M, CycA, TBP, PBGD, HTRP 1 and 18S (11-15), were investigated. The normalization strategy used was tested by analyzing the mRNA expression of B-type natriuretic peptide (BNP).

\section{Materials and methods}

Ethical approval. All animal experiments conformed to the Guide for the Care and Use of Laboratory Animals, published by the US National Institutes of Health (NIH publication no. 85-23, 1996; Bethesda, MD, USA) and were approved by the Ethics Review Board for Animal Studies of Nanjing Drum Tower Hospital (DTH ERBA 58.01/026B/2011). 
HF mouse models

MImodel. Adult male C57BL/6 mice (8 weeks old, 18-23 g, $\mathrm{n}=9$ ) were purchased from the Model Animal Research Center of Nanjing University (Nanjing, China). Mice were anesthetized by intraperitoneal administration of a mixture of ketamine hydrochloride $(50 \mathrm{mg} / \mathrm{kg}$; Jiangsu Hengrui Medicine Co., Ltd., Lianyungang, China) and diazepam $(2.5 \mathrm{mg} / \mathrm{kg}$; Tianjin Jinyao Amino Acid Co., Ltd., Tianjin, China). To detect response to tactile stimuli, the tips of the whiskers were stroked and a forepaw was pinched slightly using atraumatic forceps. The absence of a response to painful stimuli to the tail was considered indicative of adequate anesthesia. Any reaction or movement was judged as a positive response. Mice were endotracheally intubated and mechanically ventilated using a $16 \mathrm{G}$ needle (Finlumen, Inc., Beijing, China), which was inserted $\sim 2 \mathrm{~cm}$ behind the tongue of the mice (tidal volume, 6-8 ml/kg; frequency, $120 \mathrm{bpm}$; respiration ratio, 1:1; Jiangxi Teli Anaesthesia \& Respiration Equipment Co., Ltd., Nanchang, China) with supplemental oxygen. The heart was exposed through a thoracotomy on the left at the third or fourth intercostal space, and the left anterior descending coronary artery (LAD) was ligated with a 7-0 silk suture, $1 \mathrm{~mm}$ below the tip of the left atrial appendage. MI was confirmed by myocardial blanching and electrocardiographic observations. The mice of the sham group $(n=9)$ underwent a thoracotomy and pericardiotomy without LAD ligation. Prior to sacrifice, the cardiac function of the mice was evaluated by transthoracic ultrasonic cardiogram (UCG) to confirm the successful creation of the HF models. The mice were anesthetized again by ketamine hydrochloride $(50 \mathrm{mg} / \mathrm{kg})$ and diazepam $(2.5 \mathrm{mg} / \mathrm{kg})$, and sacrificed by intravenous administration of $2 \mathrm{~mol} / \mathrm{l} \mathrm{KCl}$. The hearts were collected for further analyses.

TAC model. C57BL/6 male mice ( 8 weeks old, 18-23 g, $\mathrm{n}=10$ ) were anesthetized as described above. The chest cavity was entered through the second intercostal space at the left upper sternal border via a small incision. Using a light source with two flexible fiber optic arms, the thymus was gently moved from the field of view to expose the aortic arch. The transverse aorta was isolated between the carotid arteries, and then it was constricted by a 7-0 silk suture ligature that was tied firmly against a 27-gauge needle (Becton Dickinson Co., Franklin Lakes, NJ, USA). The latter was promptly removed to yield a constriction of $0.4 \mathrm{~mm}$ in diameter. The chest was closed with a 5-0 silk suture (Shanghai Pudong Jinhuan medical products Co., Ltd., Shanghai, China), and the mice were allowed to recover from the anesthesia with an external heat supply to maintain body temperature at $37^{\circ} \mathrm{C}$. The surgical preparation was performed without the use of a microscope and lasted $30 \mathrm{~min}$.

$U C G$. The M-mode measurements of the left ventricular (LV) dimensions were averaged from $>3$ cycles. LV end-systolic diameters (LVESD) and end-diastolic diameters (LVEDD) were measured. The percentage LV fractional shortening $(\mathrm{FS} \%)$ and percentage ejection fraction $(\mathrm{EF} \%)$ were calculated as follows: FS\% $=($ LVEDD-LVESD $) /$ LVEDD x 100; and $\mathrm{EF} \%=\mathrm{EF} \%=100 \times\left[\left((7.0 /(2.4+\mathrm{LVEDD}))^{*}\right.\right.$ LVEDD $^{3}-\left(\left(7.0 /\left(2.4+\right.\right.\right.$ LVESD $\left._{)}\right) *$ LVESD $\left.^{3}\right) /\left(\left(7.0 /\left(2.4+\right.\right.\right.$ LVEDD $\left._{)}\right) *$ $\left.\mathrm{LVEDD}^{3}\right]$

Histological examination. Hearts from mice at 4 weeks following MI and 8 weeks following TAC were fixed with

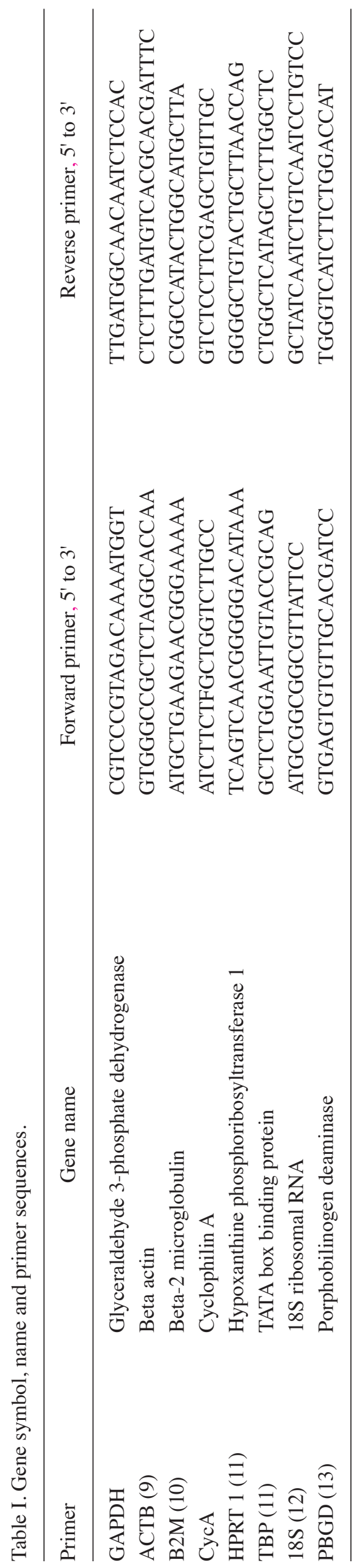


A

$\underline{3 \mathbf{m m}}$

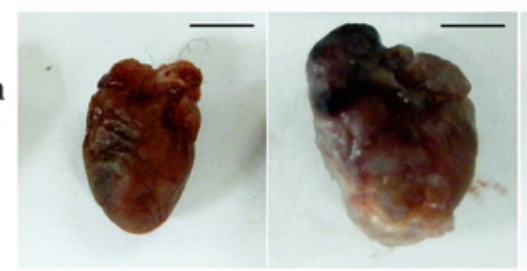

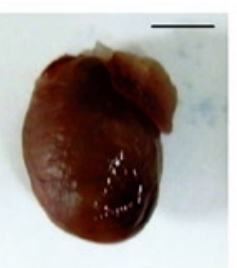

B

$3 \mathrm{~mm}$
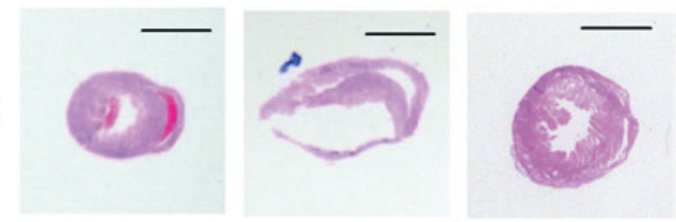

C

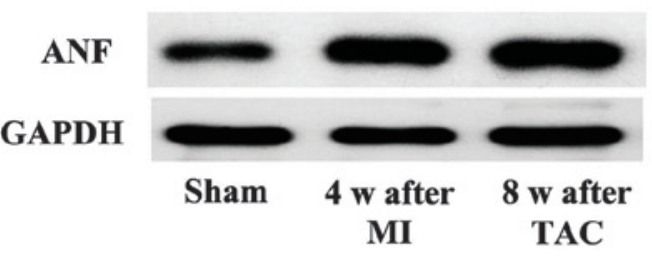

D

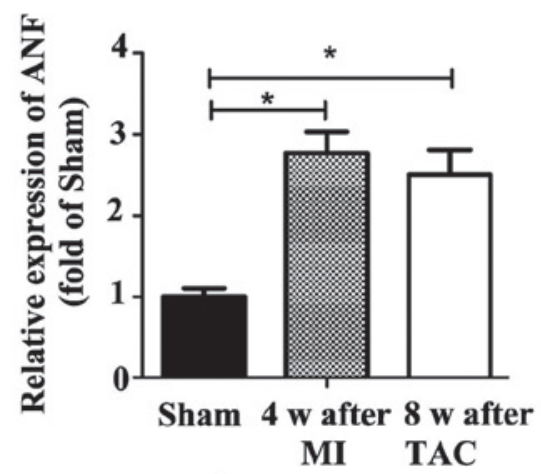

E

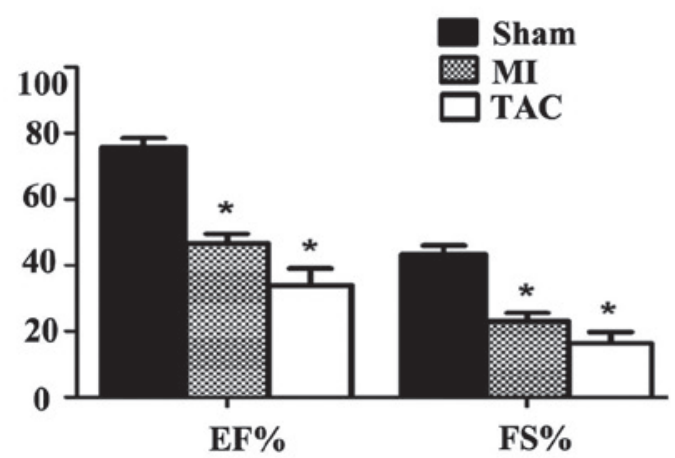

F

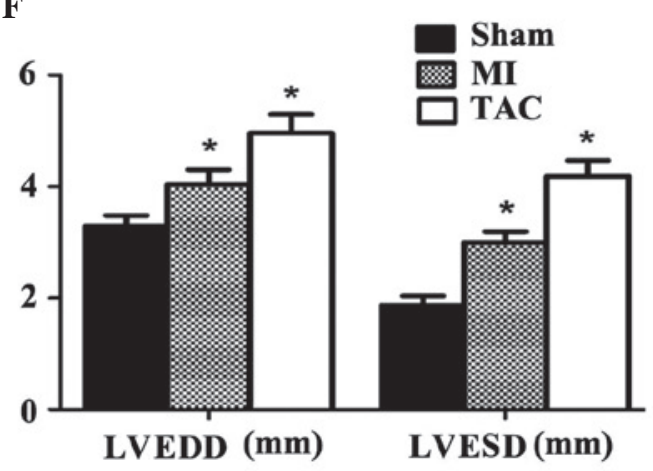

Figure 1. Genration of two mouse models of heart failure. (A) Representative images of hearts from the MI model (middle, $\mathrm{n}=3$ ) and TAC model (right, $\mathrm{n}=3$ ). The MI and TAC hearts appear more spherical as compared with the hearts of the sham group (left, $\mathrm{n}=3$ ). The scale bar $=3$ mm. (B) Paraffin-embedded cross sections of the papillary muscles of myocardium were stained with hematoxylin and eosin in the MI (middle), TAC (right) and sham (left) groups. The scale bar $=3$ mm. (C and D) Western blot analysis of ANF expression. Protein extracts were collected from the left ventricles of the mice. ANF expression was higher in the MI and TAC groups as compared with the sham group. (E and F) MI and TAC group exhibited lower EF\% and FS\%, but greater LVEDD and LVESD ("P<0.05, vs. sham group). MI, myocardial infarction; TAC, transverse aortic arch constriction; ANF, atrial natriuretic factor; EF, ejection fraction; FS, fractional shortening; LVEDD, left ventricular end diastolic diameter; LVESD, left ventricular end systolic diameter.

formalin, embedded with paraffin (Beijing Zhongshan Golden Bridge Biotechnology Co., Ltd., Beijing, China) and cut into $4 \mathrm{~mm}$ slices for histological analysis. The sizes of the hearts were assessed by histological analysis with hematoxylin and eosin staining.

$R N A$ isolation and reverse transcription $(R T)$. Total RNA was extracted using TRIzol ${ }^{\circledR}$ (Invitrogen Life Technologies, Carlsbad, CA, USA) according to the manufacturer's instructions. The ratio of the optical densities at 260 and $280 \mathrm{~nm}$ were used to assess the quality and quantity of the total RNA. The left ventricle of the heart was chosen for qPCR analysis. cDNA was prepared from $1 \mu \mathrm{g}$ total RNA using the PrimeScript RT reagent kit (Takara Biotechnology Co., Ltd., Dalian, China). The Oligo dT Primer from the kit was used for the RT reaction, and the cDNA was stored at $-80^{\circ} \mathrm{C}$ until further use.

qPCR. The sequences of primers used for PCR are listed in Table I. The reactions contained $2 \mathrm{X} \mathrm{SYBR}^{\circledR}$ Premix Ex Taq,
50X Rox Reference Dye II, forward and reverse primers (200 $\mathrm{nM}$ ) and $2 \mu \mathrm{l}$ cDNA template, in a $20 \mu \mathrm{l}$ reaction volume. The amplification was performed with an initial denaturation step at $95^{\circ} \mathrm{C}$ for $30 \mathrm{sec}$, followed by 40 cycles of denaturation at $95^{\circ} \mathrm{C}$ for $5 \mathrm{sec}$, annealing and extension at $60^{\circ} \mathrm{C}$ for $34 \mathrm{sec}$. Following amplification, the specificity was confirmed by melting-curve analysis of the PCR products. The qPCR was performed using the Applied Biosystems 7500 Real-Time PCR device. All of the samples were run in triplicate.

Analysis of $R G$ expression stability. geNorm is an appropriate selection for the analysis of the stability of RGs. It makes use of the comparative cycle threshold $(\mathrm{Ct})$-method to validate the stability of RG RNA expression levels. The lowest $\mathrm{Ct}$ value was set to a comparative expression level of 1 , and the expression levels of the same RGs were relatively quantified in accordance with the $2^{-\Delta \Delta C t}$ method. The geNorm tool was also used to calculate the stability measures (M) of the RGs. The genes were ranked by their $M$ value, and at each step of the analysis the least 
Table II. Comparison of expression stability ( $\mathrm{M}$ value) of RGs during step-wise exclusion of the least stable gene in sham group, $\mathrm{MI}+$ sham group, $\mathrm{TAC}+$ sham group and TAC + MI + sham group.

\begin{tabular}{|c|c|c|c|c|c|c|c|}
\hline \multicolumn{2}{|l|}{ Sham } & \multicolumn{2}{|c|}{ MI + sham } & \multicolumn{2}{|c|}{ TAC + sham } & \multicolumn{2}{|c|}{$\mathrm{TAC}+\mathrm{MI}+$ sham } \\
\hline Gene & M & Gene & M & Gene & M & Gene & M \\
\hline HPRT 1 & 2.21 & HPRT 1 & 1.31 & HPRT 1 & 1.02 & HPRT 1 & 1.22 \\
\hline $18 \mathrm{~S}$ & 1.45 & B2M & 1.06 & $18 \mathrm{~S}$ & 0.63 & $18 \mathrm{~S}$ & 1.00 \\
\hline СусA & 1.09 & TBP & 1.08 & TBP & 0.51 & B2M & 0.86 \\
\hline ACTB & 0.79 & $18 \mathrm{~S}$ & 0.61 & GAPDH & 0.42 & TBP & 0.71 \\
\hline TBP & 0.71 & ACTB & 0.34 & CycA & 0.20 & GAPDH & 0.49 \\
\hline $\mathrm{B} 2 \mathrm{M}$ & 0.57 & PBGD & 0.31 & PBGD & 0.18 & СycA & 0.26 \\
\hline PBGD/GAPDH & 0.27 & CycA/GAPDH & 0.02 & В2M/АCТВ & 0.00 & PBGD/ACTB & 0.13 \\
\hline
\end{tabular}

A

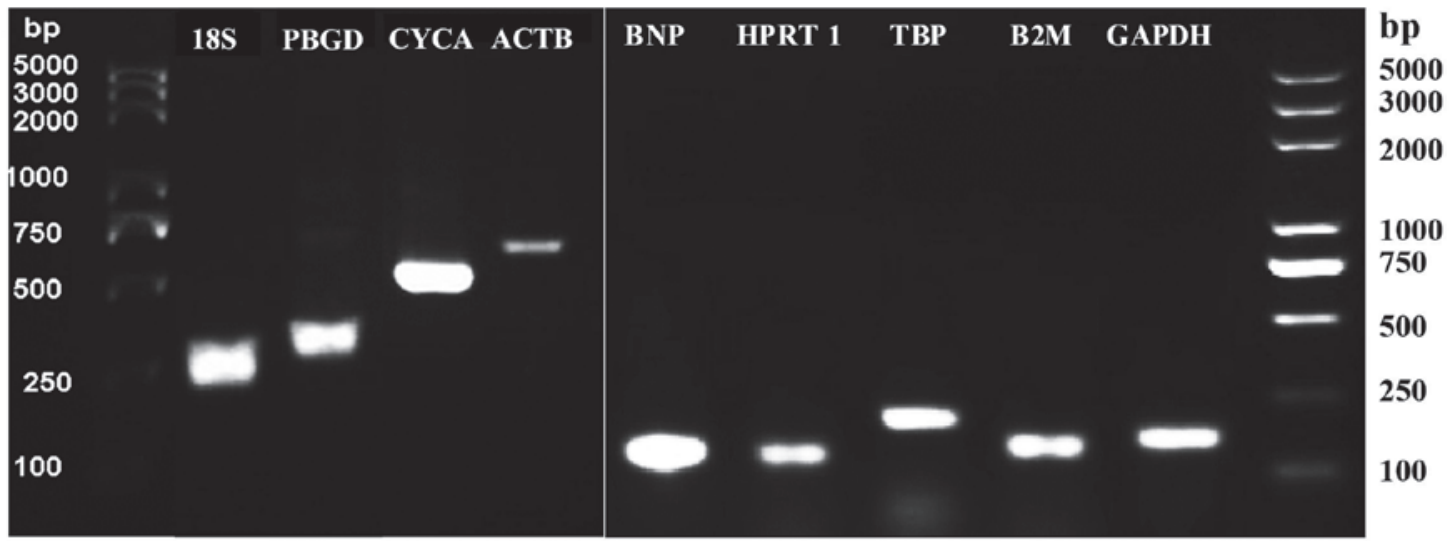

B

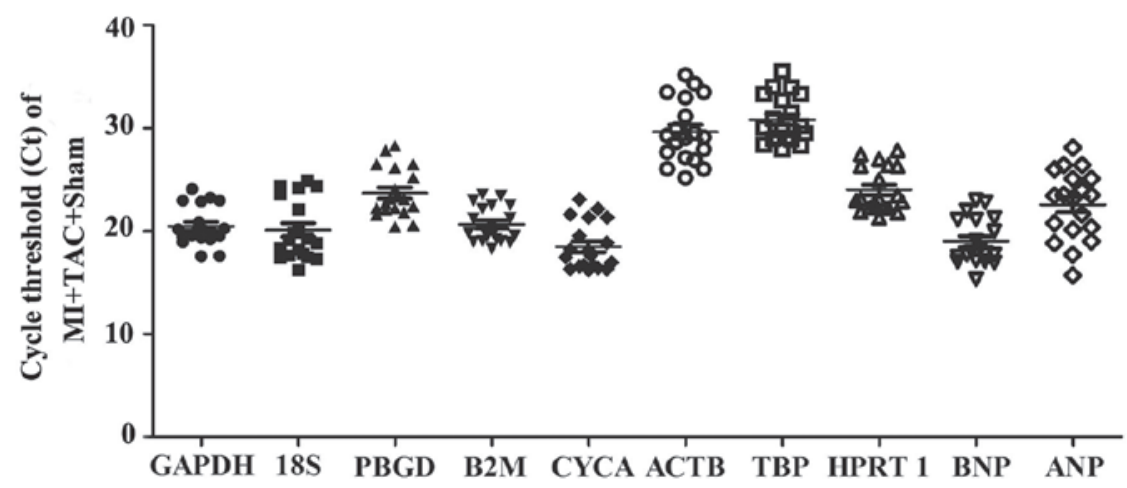

Figure 2. Primer specificity and efficiency. (A) Agarose gel electrophoresis of polymerase chain reaction products. (B) Cycle threshold (Ct) distribution of each putative reference gene (RG) in the samples. The Ct was determined for each RG tested and the distribution of the RGs in the 19 samples obtained from the 3 groups was determined.

stable gene (highest $\mathrm{M}$ value) was excluded and $\mathrm{M}$ was recalculated. The most stable pair of genes was identified through the reiteration of this procedure in a step-wise manner. The optimal number of genes for normalization was determined as previously described (4), starting from the most stable pair and sequentially adding the other genes (from the most to least stable) until the pair-wise variation reached a set threshold (usually, M-value $<1.5$ and pair-wise variation $<0.15$ ).

Statistical analysis. SPSS software, version 13.0 (SPSS, Inc., Chicago, IL, USA) was used for statistical analysis. In all cases, the data were compared by one-way analysis of variance.
$\mathrm{P}<0.05$ was considered to indicate a statistically significant difference.

Western blot analysis. Proteins were extracted from the left ventricles of the hearts and assessed by western blotting. The primary antibodies used were rabbit anti-GAPDH poltclonal antibody targeting human, mouse and rat (AP 0066; Bioworld Technology, Inc., St. Louis Park, MN, USA) and rabbit anti-ANF targeting human, mouse and dog (ab91250; Abcam, Cambridge, MA, USA). Phosphate-buffered saline containing $8.0 \mathrm{~g} \mathrm{NaCl}$, $0.2 \mathrm{~g} \mathrm{KCl}, 1.44 \mathrm{~g} \mathrm{Na}_{2} \mathrm{HPO}_{4}, 0.24 \mathrm{~g} \mathrm{KH}_{2} \mathrm{PO}_{4}$ and $\mathrm{ddH}_{2} \mathrm{O}$ to $1000 \mathrm{ml}$ (pH 7.4) was used as a dilution for the antibodies Remote 


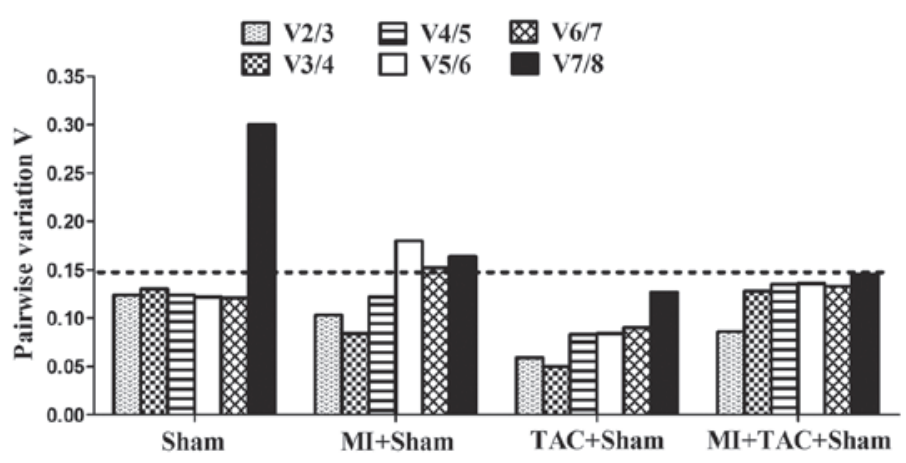

Figure 3. Pair-wise variation analysis between sequential normalization factors. The pairwise variation coefficient, calculated between the sequential normalization factors, is used to determine of the optimal number of reference genes (RGs). V2/3 indicates the change in stability when using 3 instead of 2 RGs to calculate the normalization factor. A pair-wise variation coefficient of $\leq 0.15$ was regarded as an appropriate cut-off. Therefore, a combination of 2 RGs is appropriate to use in each sample subset. The increasing values are due to the inclusion of unstable RGs. MI, myocardial infarction; TAC, transverse aortic arch constriction.

A

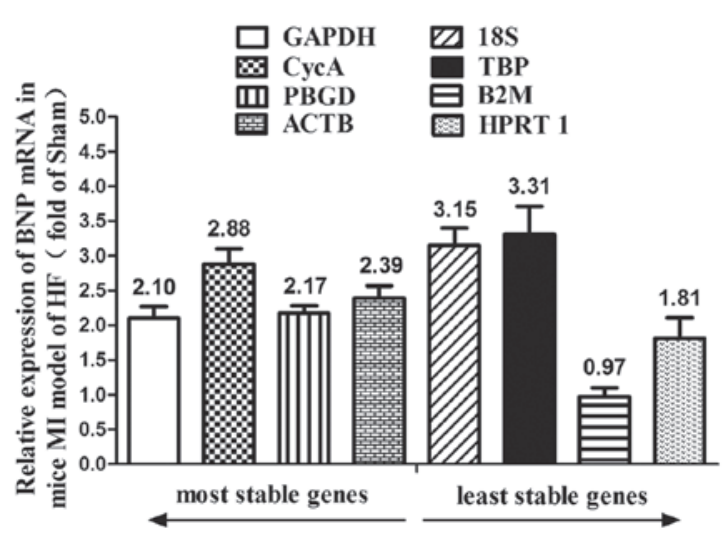

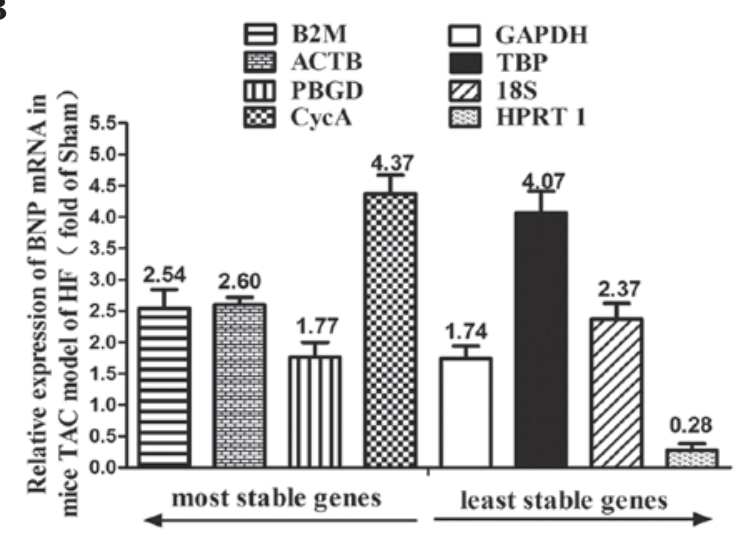

Figure 4. BNP mRNA expression in the left ventricle. The relative expression levels of BNP in the left ventricles of (A) MI group (n=6) and (B) TAC group $(n=7)$ (expressed as fold of the sham group, $n=6)$. HF, heart failure; MI, myocardial infarction; TAC, transverse aortic arch constriction.

myocardium was homogenized with a Dounce tissue grinder in lysis buffer consisting of: $10 \mathrm{mM}$ Tris/ $\mathrm{HCl}$ (pH 7.2), $150 \mathrm{mM}$ $\mathrm{NaCl}, 0.1 \%$ SDS, $1 \%$ (v/v) Triton X-100, $1 \%$ sodium deoxycholate, $5 \mathrm{mM}$ EDTA, $1 \mathrm{mM} \mathrm{Na} \mathrm{VO}_{4}, 50 \mathrm{mM} \mathrm{NaF}$ (all Sigma), $0.2 \mathrm{mM}$ phenymethylsulfonyl fluoride and protease inhibitor cocktail (complete EDTA-free; Roche Diagnostics, Basel, Switzerland). Protein content was determined by a bicinchoninic acid protein assay kit (Pierce Biotechnology, Inc., Rockford, IL, USA). Proteins were separated by SDS-polyacrylamide gel electrophoresis and transferred to Immobilon ${ }^{\mathrm{TM}}$ polyvinylidene fluoride membranes (EMD Millipore, Billerica, MA, USA).

\section{Results}

HF in mouse models following TAC and MI. Four and eight weeks subsequent to $\mathrm{MI}$ and TAC, respectively, there was a marked cardiac enlargement (Fig. 1A and B). To confirm the extent of damage to the heart, the expression levels of $\mathrm{HF}$ markers were determined (16). The western blot analysis of ANF indicated a reduction of heart function in the HF mouse models (Fig. 1C and D). UCG analysis also revealed left ventricle enlargement, dilatation and systolic dysfunction 4 weeks and 8 weeks subsequent to $\mathrm{MI}$ and TAC, respectively (Fig. 1E and F). These findings indicated the successful production of mouse models of HF.
Primer specificity and efficiency. The hearts of mice in the two model groups were resected and analyzed. Following amplification of the primers, the expression levels of the RGs were observed by $1.5 \%$ agarose gel electrophoresis (Power Pac 200; Bio-Rad, Hercules, CA, USA). As presented in Fig. 2A, the bands of the RGs, including GAPDH, ACTB, B2M, CycA, TBP, PBGD, HTRP 1 and 18S, were clear. The melting-curve analysis of the eight RGs during the qPCR revealed a single product with a melting temperature in accordance with the expected value (data not shown). The distributions of $\mathrm{Ct}$ data among the eight RGs and BNP covered a wide range, from 16 to 34 cycles in the MI, sham and TAC groups (Fig. 2B).

Analysis of RGs using geNorm software. geNorm was used for the analysis of specimens from the sham, MI and TAC groups. A lower M-value indicated a more stable gene expression. All RGs, except HPRT 1 presented an M-value below the theoretical threshold of 1.5 in the sham group. In the sham group, the most stably expressed genes were PBGD and GAPDH. When combined with MI or TAC specimens, the most stable RGs were CycA and GAPDH or B2M and ACTB, respectively. In the $\mathrm{MI}+\mathrm{TAC}+$ sham group, the most stable RGs were PBGD and ACTB (Table II). In all the tissue subsets, the combination of the two best RGs was identified to be sufficient for gene normalization with pair-wise variation values of $\leq 0.15$ (Fig. 3 ). 
BNP mRNA expression. BNP expression was analyzed to assess whether the normalization strategy had been effectively applied (17). The mRNA levels of BNP were normalized to the subset of selected RGs. As presented in Fig. 4, the relative BNP mRNA levels differed when normalized to each different selected RGs. With the exception of B2M, BNP expression was relatively higher in the $\mathrm{MI}$ and TAC models as compared with the sham group when normalized to the expression levels of the remaining seven RGs. qPCR analysis was consistent with geNorm software analysis. The data suggest that neither B2M or HPRT 1 should be used as RGs in the MI model, nor HPRT 1 in the TAC model of HF.

\section{Discussion}

The current study aimed to illustrate the variations in the stability of RGs in mouse models of MI and TAC of HF. To the best of our knowledge, the present study is the first to describe the selection of RGs in C57BL/6 mice with two different models of HF. A combination of PBGD and ACTB was determined to be best for use as RGs in mouse $\mathrm{MI}+\mathrm{TAC}+$ sham models of HF. In addition, a combination of GAPDH and CycA were identified as the most stable RGs for use in the MI model, while B2M and ACTB were most appropriate in the TAC model. The use of HPRT 1 as an RG in C57BL/6 mice was not indicated to be appropriate for use in the mouse models generated in the present study.

The C57BL/6 mouse is widely used to identify potentially important and novel targets for pharmacological or molecular therapy for HF. The mouse HF models not only provide data during the development of the chronic changes in myocardial structure and function, but also quantify the progression of HF and left ventriclar dysfunction. The MI $(18,19)$ and TAC $(19,21)$ mouse models are the most widely and successfully used HF models.

The expression of eight commonly used RGs, including GAPDH (5), ACTB, B2M, CycA (6,22), TBP (6), PBGD (23), HTRP 1 (6) and 18S (1), were analyzed to determine which is the best set for use in the mouse models. The experimental protocol consisted of an LV, which was able to produce a marked remodeling and dilation of the heart as a HF model (24). Substantial differences in gene expression stability, expressed as M-values, were observed between the mouse models. It may be interpreted that during HF, there are different specific physiological and functional changes involved in the different models.

CycA has been used as an RG in the atrium and right ventricle of the minipig HF model (induced by rapid LV pacing for 180 beats $/ \mathrm{min}$ ) (6). However, other studies have indicated that CycA, which is secreted by inflammatory and dying/necrotic cells, was significantly enhanced in patients with inflammatory cardiomyopathy (22) and was able to promote atherosclerosis in mice (25). CycA was determined as one of the most stable RGs in the MI mouse model of HF in the present study, but it was not conclusive whether the mice developed atherosclerosis.

A previous study indicated that GAPDH could not be used as an RG as its expression was highly variable in MI mouse tissues (26). In particular, GAPDH was also demonstrated to be the optimal RG in a rat MI model of HF (2). The results of the present study indicated that GAPDH, which is commonly used for gene expression normalization in MI studies, presented the least expression variability in tissues of the MI model mice, and in combination with CycA created the best set of RGs for the MI mouse model.

ACTB, a cytoskeletal protein, has been indicated to be the most stable gene in heart tissues from murine models of cardiometabolic disease (produced by feeding with high-fat diet) (8). Yperman et al (27) demonstrated that ACTB was not stable in certain types of interstitial cell, thus it cannot be regarded as a reference for gene expression of ovine-derived heart valve interstitial cells in culture. In the current study, ACTB, combined with B2M or PBGD, was observed to be the best RG for the TAC or TAC + MI model.

In order to confirm the results from geNorm, the relative expression of BNP, a 32-amino acid polypeptide secreted by the ventricles of the heart in response to excessive stretching of cardiomyocytes (28), was evaluated. The results displayed a marked difference in the levels of BNP mRNA normalized to the set of the selected RGs. Therefore, the upregulation of BNP mRNA expression was in agreement with the data of the study by Bhalla et al (28), and the comparison revealed that the levels may vary significantly, depending on the RGs used for normalization.

In summary, the present study identified and validated a set of stably expressed RGs for use in mouse MI and TAC models of HF. The results indicated that GAPDH combined with CycA or B2M with ACTB were the best set of RGs in MI or TAC mouse models, respectively. Where MI and TAC mouse models are required simultaneously, it is preferred to use ACTB and PBGD as the RGs. The current study provides important information for RG selection, and will facilitate further investigations into the transcriptome of mice with aberrant heart function.

\section{Acknowledgements}

This study was supported by grants from the Natural Science Foundations of China (grant nos. 81071859, $81000055,81200148,81270281$ and 81200092), the Jiangsu Key Laboratory for Molecular Medicine of Nanjing University (grant no. 2008), the Jiangsu Provincial Special Program of Medical Science (grant no. BL2012014), the State Key Laboratory of Pharmaceutical Biotechnology (grant no. KF-GN-200901), the Peak of Six Personnel in Jiangsu Province (grant no. 2013-WSN-008) and the Funds for Distinguished Young Scientists in Nanjing (Xie Jun).

\section{References}

1. Wang JX, Jiao JQ, Li Q et al: miR-499 regulated mitochondrial dynamics by targeting calcineurin and dynamin-related protein-1. Nat Med 17: 71-78, 2011.

2. Brattelid T, Tveit K, Birkeland JA, et al: Expression of mRNA encoding $\mathrm{G}$ protein-coupled receptors involved in congestive heart failure - a quantitative RT-PCR study and the question of normalisation. Basic Res Cardiol 102: 198-208, 2007.

3. de Jonge HJ, Fehrmann RS, de Bont ES, et al: Evidence based selection of housekeeping genes. PLoS One 2: e898, 2007.

4. Schmittgen TD and Zakrajsek BA: Effect of experimental treatment on housekeeping gene expression: validation by real-time quantitative RT-PCR. J Biochem Biophys Methods 46: 69-81, 2000.

5. Sundaresan NR, Vasudevan P, Zhong L, et al: The sirtuin SIRT6 blocks IGF-Akt signaling and development of cardiac hypertrophy by targeting c-Jun. Nat Med 18: 1643-1650, 2012. 
6. Martino A, Cabiati M, Campan M, et al: Selection of reference genes for normalization of real-time PCR data in minipig heart failure model and evaluation of TNF- $\alpha$ mRNA expression. J Biotechnol 153: 92-99, 2011.

7. Koyanagi T, Wong LY, Inagaki K, Petrauskene OV and Mochly-Rosen D: Alteration of gene expression during progression of hypertension-induced cardiac dysfunction in rats. Am J Physiol Heart Circ Physiol 295: H220-H226, 2008.

8. Bruce KD, Stokes AJ, Patel NR, et al: Identification of robust cardiac reference genes in a mouse model of cardiometabolic disease. J Clinic Experiment Cardiol 2: 161, 2011.

9. Pilbrow AP, Ellmers LJ, Black MA, et al: Genomic selection of reference genes for real-time PCR in human myocardium. BMC Med Genomics 1: 64, 2008.

10. Vandesompele J, De Preter K, Pattyn F, et al: Accurate normalization of real-time quantitative RT-PCR data by geometric averaging of multiple internal control genes. Genome Biol 3 : RESEARCH0034, 2002.

11. Yang $\mathrm{CH}$, Ji YY and Yeh M: The kinetics of IL-4 and IFN-gamma gene expression in mice after Trichosansin immunization. Cell Res 8: 295-302, 1998.

12. Teixeira-Clerc F, Julien B, Grenard P, et al: CB1 cannabinoid receptor antagonism: a new strategy for the treatment of liver fibrosis. Nat Med 12: 671-676, 2006.

13. Shi G, Zhang Z, Feng D, et al: Selection of reference genes for quantitative real-time reverse transcription-polymerase chain reaction in concanavalin A-induced hepatitis model. Ana Biochem 401: 81-90, 2010.

14. Chen Y, Li K, Zhou YX and Xiao JH: Selection of reference genes for transcription expression analysis in the mouse. Acta Laboratorium Animalis Scientia Sinica 19: 197-202, 2011.

15. McManamny P, Chy HS, Finkelstein DI, et al: A mouse model of spinal and bulbar muscular atrophy. Hum Mol Genet 11: 2103-2111, 2002.

16. Tan SC, Carr CA, Yeoh KK, et al: Identification of valid housekeeping genes for quantitative RT-PCR analysis of cardiosphere-derived cells preconditioned under hypoxia or with prolyl-4-hydroxylase inhibitors. Mol Biol Rep 39: 4857-4867, 2012.
17. Oudit GY, Crackower MA, Eriksson U, et al: Phosphoinositide 3-kinase gamma-deficient mice are protected from isoproterenol-induced heart failure. Circulation 108: 2147-2152, 2003.

18. Hu S, Huang M, Li Z, et al: MicroRNA-210 as a novel therapy for treatment of ischemic heart disease. Circulation 122 (Suppl): S124-S131, 2010.

19. Lygate CA, Aksentijevic D, Dawson D, et al: Living without creatine: unchanged exercise capacity and response to chronic myocardial infarction in creatine-deficient mice. Circ Res 112: 945-955, 2013.

20. Anand P, Brown JD, Lin CY, et al: BET bromodomains mediate transcriptional pause release in heart failure. Cell 154: 569-582, 2013.

21. Kondo K, Bhushan S, King AL, et al: $\mathrm{H}_{2} \mathrm{~S}$ protects against pressure overload-induced heart failure via upregulation of endothelial nitric oxide synthase. Circulation 127: 1116-1127, 2013.

22. Zuern CS, MüllerKA, SeizerP, et al: Cyclophilin A predicts clinical outcome in patients with congestive heart failure undergoing endomyocardial biopsy. Eur J Heart Fail 15: 176-184, 2013.

23. Hölschermann H, Bohle RM, Schmidt H, et al: Hirudin reduces tissue factor expression and attenuates graft arteriosclerosis in rat cardiac allografts. Circulation 102: 357-363, 2000.

24. Recchia FA and Lionetti V: Animal models of dilated cardiomyopathy for translational research. Vet Res Commun 31 (Suppl 1): 35-41, 2007.

25. Nigro P, Satoh K, O'Dell MR, et al: Cyclophilin A is an inflammatory mediator that promotes atherosclerosis in apolipoprotein E-deficient mice. J Exp Med 208: 53-66, 2011.

26. Everaert BR, Boulet GA, Timmermans JP and Vrints CJ Importance of suitable reference gene selection for quantitative real-time PCR: special reference to mouse myocardial infarction studies. PLoS One 6: e23793, 2011.

27. Yperman J, Visscher G, Holvoet P and Flameng W: Beta-actin cannot be used as a control for gene expression in ovine interstitial cells derived from heart valves. J Heart Valve Dis 13: 848-853, 2004.

28. Bhalla V, Willis S and Maisel AS: B-type natriuretic peptide: the level and the drug - partners in the diagnosis of congestive heart failure. Congest Heart Fail 10 (Suppl 1): 3-27, 2004. 